\title{
Mesolimbic opioid-dopamine interaction is disrupted in obesity but recovered by weight loss following bariatric surgery
}

Henry K. Karlsson (1)', Lauri Tuominen (10 ${ }^{1,2}$, Semi Helin ${ }^{1}$, Paulina Salminen $\mathbb{1}^{3,4}$, Pirjo Nuutila, ${ }^{1,5}$ and Lauri Nummenmaa (D)

\begin{abstract}
Obesity is a growing burden to health and the economy worldwide. Obesity is associated with central $\mu$-opioid receptor (MOR) downregulation and disruption of the interaction between $M O R$ and dopamine $D_{2}$ receptor $\left(D_{2} R\right)$ system in the ventral striatum. Weight loss recovers MOR function, but it remains unknown whether it also recovers aberrant opioid-dopamine interaction. Here we addressed this issue by studying 20 healthy non-obese and 25 morbidly obese women (mean $\mathrm{BMI} 41$ ) eligible for bariatric surgery. Brain MOR and $\mathrm{D}_{2} \mathrm{R}$ availability were measured using positron emission tomography (PET) with $\left[{ }^{11} \mathrm{C}\right]$ carfentanil and $\left[{ }^{11} \mathrm{C}\right]$ raclopride, respectively. Either Roux-en-Y gastric bypass or sleeve gastrectomy was performed on obese subjects according to standard clinical treatment. 21 obese subjects participated in the postoperative PET scanning six months after bariatric surgery. In the control subjects, MOR and $\mathrm{D}_{2} \mathrm{R}$ availabilities were associated in the ventral striatum $(r=.62)$ and dorsal caudate $(r=.61)$. Preoperatively, the obese subjects had disrupted association in the ventral striatum $(r=.12)$ but the unaltered association in dorsal caudate $(r=.43)$. The association between MOR and $\mathrm{D}_{2} \mathrm{R}$ availabilities in the ventral striatum was recovered $(r=.62)$ among obese subjects following the surgery-induced weight loss. Bariatric surgery and concomitant weight loss recover the interaction between MOR and $D_{2} R$ in the ventral striatum in the morbidly obese. Consequently, the dysfunctional opioid-dopamine interaction in the ventral striatum is likely associated with an obese phenotype and may mediate excessive energy uptake. Striatal opioid-dopamine interaction provides a feasible target for pharmacological and behavioral interventions for treating obesity.
\end{abstract}

\section{Introduction}

The prevalence of obesity is dramatically increasing and there is an urgent need for novel efficient therapies. Numerous studies point towards the role of the brain in the development and maintenance of obesity ${ }^{1,2}$. Previous studies indicate that both opioid and dopamine systems in the brain's reward circuit are dysfunctional in obesity. The endogenous opioid system has been linked to hedonic aspects of feeding in animals $s^{3,4}$. In humans, both $\mu$-opioid

\footnotetext{
Correspondence: Henry K. Karlsson (hekrka@utu.fi)

${ }^{1}$ Turku PET Centre, Turku University Hospital and University of Turku, Turku, Finland

${ }^{2}$ Institute of Mental Health Research, University of Ottawa, Ottawa, ON, Canada Full list of author information is available at the end of the article
}

receptor (MOR) antagonists and inverse agonists have been shown to reduce eating behavior ${ }^{5,6}$. Previously, decreased MOR availability has been observed in the reward circuit among obese subjects ${ }^{7,8}$. Thus, aberrant opioid functioning in obesity may diminish the opioiddependent rewarding effects of eating. Alterations in dopamine $D_{2}$ receptor $\left(D_{2} R\right)$ expression and function in obesity have been observed in some ${ }^{9-12}$ but not all human imaging studies ${ }^{8,13}$. It is possible that the relationship between $D_{2} R$ availability and measures of obesity is not linear, but quadratic ${ }^{14}$ or age-dependent ${ }^{15}$. Alternatively, it is possible that the effects of obesity on D2R are mediated via the MOR system. 
Tight interaction between dopaminergic and opioidergic systems has been proposed to underlie human reward functions ${ }^{16}$, but only a few studies have actually investigated this issue. In humans, dopamine-releasing drugs such as cocaine and amphetamine lead to endogenous opioid release ${ }^{17-19}$. In rats, both $\mathrm{D}_{2}$ Rs and MORs are closely connected in the striatum, which can be morphologically divided into striosome/patch and matrix compartments. MORs can control the release of dopamine by inhibiting GABAergic interneurons in VTA $^{20-25}$. Furthermore, VTA dopamine neurons express MOR postsynaptically, and direct inhibition between MOR and dopamine neurons exists without GABAergic signaling ${ }^{26}$.

Cross-talk between opioidergic and dopaminergic systems may underlie aberrant reward-related behaviors, such as excessive feeding. In rats, intravenous administration of MOR agonists triggers dopamine release and feeding $^{27}$, while MOR antagonists block dopamine release and reduce food consumption ${ }^{28}$. Finally, in vivo PET data from humans show that there is a close interaction between $M O R$ and $D_{2} R$ receptors in the reward circuit among non-obese subjects, while this interaction is disrupted in the ventral striatum among obese subjects, potentially contributing to obesity ${ }^{29}$. However, it remains unclear whether the dysfunctional $M O R / D_{2} R$ interaction reflects a vulnerability endophenotype for obesity, or whether it develops as a consequence of the obese state.

Bariatric surgery is the most effective method for weight loss in obesity ${ }^{30}$. The surgical procedure significantly lowers appetite $^{31}$, but the actual molecular brain mechanisms behind this are still poorly understood. Bariatric surgery provides a powerful method for investigating changes in neuroreceptor systems and opioid-dopamine interaction after weight gain. Previous studies have investigated the effects of bariatric surgery and following weight loss to separate receptor systems, showing mainly unaltered $D_{2} R$ availability and normalized MOR availability ${ }^{32-35}$. Here we tested whether bariatric surgery-induced weight loss could recover the dysfunctional opioid-dopamine interaction in the obese.

\section{Subjects and methods}

The study was conducted in accordance with the Declaration of Helsinki and approved by the Ethical Committee of the Hospital District of South-Western Finland (SleevePET2, NCT01373892, http://www.clinicaltrials.gov). All participants gave a signed informed consent form prior to scans.

\section{Subjects}

We recruited 25 morbidly obese women (mean BMI $41 \mathrm{~kg} / \mathrm{m}^{2}$ ) eligible for bariatric surgery. Either Roux-en-Y gastric bypass or sleeve gastrectomy was performed as their standard clinical treatment. Four subjects discontinued the study for personal reasons, and 21 subjects participated in the postoperative scanning six months after the surgical procedure. 20 non-obese healthy women (mean BMI $22 \mathrm{~kg} / \mathrm{m}^{2}$ ) formed the control group. Data for this patient cohort have been reported previously ${ }^{8,29,34}$. The sample size was determined by a priori power analysis based on our previous studies ${ }^{34}$. Characteristics of the subjects are presented in Table 1. Clinical screening of the subjects included history, physical examination, anthropometric measurements, and laboratory tests. Exclusion criteria involved opiate drug use, binge-eating disorders, neurological and severe mental disorders, substance abuse, excessive alcohol consumption (more than eight units per week) determined by clinical interviews, medical history, and blood tests. None of the controls smoked tobacco, but 8 obese subjects were smokers (3-15 cigarettes per day). Antidiabetic, antihypertensive, and cholesterol-lowering drugs were paused prior to the study.

\section{Image acquisition and quantification of receptor availability}

We measured $\mathrm{D}_{2}$ receptor availability with the antagonist $\left[{ }^{11} \mathrm{C}\right]$ raclopride $^{36}$ and $\mu$-opioid receptor availability

Table 1 Characteristics of the subjects.

\begin{tabular}{llll}
\hline & Obese preoperative $(\boldsymbol{N}=\mathbf{2 5})$ & Obese postoperative $(\boldsymbol{N}=\mathbf{2 1})$ & Healthy control subjects $(\boldsymbol{N}=\mathbf{2 0})$ \\
\hline Age $(\mathrm{y})$ & $41.2 \pm 9.2$ & - & $42.0 \pm 13.2$ \\
$\mathrm{BMl}\left(\mathrm{kg} / \mathrm{m}^{2}\right)$ & $41.3 \pm 4.1$ & $31.9 \pm 4.4$ & $22.4 \pm 2.6$ \\
Percentage of fat (\%) & $50.3 \pm 6.7$ & $43.2 \pm 4.2$ & $30.6 \pm 6.4$ \\
Tobacco smokers/non-smokers $(\mathrm{N})$ & $8 / 17$ & $5 / 16$ & $0 / 20$ \\
Amount of alcohol use (units per week) & $1.7 \pm 1.8$ & $\mathrm{~N} / \mathrm{A}$ & $2.9 \pm 2.3$ \\
Injected activity of $\left[{ }^{11} \mathrm{C}\right]$ carfentanil $(\mathrm{MBq})$ & $253.2 \pm 11.6$ & $252.1 \pm 15.0$ & $251.2 \pm 8.4$ \\
Injected activity of $\left[{ }^{11} \mathrm{C}\right]$ raclopride $(\mathrm{MBq})$ & $247.9 \pm 20.8$ & $254.5 \pm 10.9$ & $258.3 \pm 15.7$ \\
\hline
\end{tabular}

Data are presented as mean \pm SD. 
with the high-affinity agonist $\left[{ }^{11} \mathrm{C}\right]$ carfentanil ${ }^{37}$ using positron emission tomography (PET) on two separate visits. Subjects were scanned again with both radiotracers six months after bariatric surgery. Radiotracer production has been described previously ${ }^{8}$. Both radioligands had high radiochemical purity ( $>99 \%)$. Before scanning, a catheter was placed in the subject's left antecubital vein for tracer administration. The Head was strapped to the scanner table in order to prevent head movement. Subjects fasted two hours prior to scanning. A CT scan was performed to serve as an attenuation map. The clinical well-being of subjects was monitored during the scanning.

We injected both tracers as a bolus in separate scans on separate days. Injected amounts of $\left[{ }^{11} \mathrm{C}\right]$ carfentanil and $\left[{ }^{11} \mathrm{C}\right]$ raclopride are presented in Table 1 . After injection, radioactivity in the brain was measured with the GE Healthcare Discovery ${ }^{\mathrm{TM}} 690$ PET/CT scanner (General Electric Medical Systems, Milwaukee, WI, USA) for $51 \mathrm{~min}$, using 13-time frames. MR imaging was performed with Philips Gyroscan Intera 1.5T CV Nova Dual scanner to exclude structural abnormalities and to provide anatomical reference images for the PET scans. Anatomical images $\left(1 \mathrm{~mm}^{3}\right.$ voxel size) were acquired using a T1weighted sequence (TR $25 \mathrm{~ms}$, TE $4.6 \mathrm{~ms}$, flip angle $30^{\circ}$, scan time 376 s).

All alignment and coregistration steps were performed using SPM8 software (www.fil.ion.ucl.ac.uk/spm/) running on Matlab R2012a (The Mathworks Inc., Sherborn, Massachusetts). To correct for head motion, dynamic PET images were first realigned frame-to-frame. The individual T1-weighted MR images were coregistered to the summation images calculated from the realigned frames. Regions of interest (ROIs) for reference regions were drawn manually on MRI images using PMOD 3.4 software (PMOD Technologies Ltd., Zurich, Switzerland). The occipital cortex was used as the reference region for $\left[{ }^{11} \mathrm{C}\right]$ carfentanil and cerebellum for $\left[{ }^{11} \mathrm{C}\right]$ raclopride. Receptor availability was expressed in terms of $B P_{\mathrm{ND}}$, which is the ratio of specific to non-displaceable binding in the brain. $B P_{\mathrm{ND}}$ was calculated by applying the basis function method for each voxel using the simplified reference tissue model (SRTM) with reference tissue time-activity curves (TAC) as input data ${ }^{38}$.

The subject-wise parametric $B P_{\mathrm{ND}}$ images were normalized to the MNI space using the T1-weighted MR images and smoothed with a Gaussian kernel of $8 \mathrm{~mm}$ FWHM. Anatomic regions of interest were generated in the ventral striatum, dorsal caudate nucleus, and putamen using the $\mathrm{AAL}^{39}$ and Anatomy ${ }^{40}$ toolboxes. Statistical analysis was performed as described earlier ${ }^{29}$. In the ROI analysis, Pearson correlation was calculated between the tracer-wise $B P_{\mathrm{ND}} \mathrm{S}$ in the striatal regions of interest. Fisher's $z$-test was used for quantifying whether ROI-level Pearson correlations between the $\left[{ }^{11} \mathrm{C}\right]$ raclopride and $\left[{ }^{11} \mathrm{C}\right]$ carfentanil $B P_{\mathrm{ND}}$ values were statistically different between groups. Normality assumption was tested with the Kolmogorov-Smirnov test.

To determine the striatal volumes in all three groups, we used T1 images and automated FreeSurfer volumetric analysis (Version 7). Preoperative differences in striatal volumes between obese and non-obese subjects were quantified with independent samples $\mathrm{t}$-test and after bariatric surgery in the obese subjects using repeatedmeasures $t$-test.

\section{Results}

MOR and $D_{2} R$ availabilities are presented in the supplementary table ( $\mathrm{S} 1)$. MOR and $\mathrm{D}_{2} \mathrm{R}$ availabilities were associated in the ventral striatum $(r=.62, p<0.05)$ and dorsal caudate $(r=.61, p<0.05)$ in the control subjects (Fig. 1). Preoperatively, the obese subjects had disrupted association in the ventral striatum $(r=.12$, ns), but the unaltered association in dorsal caudate $(r=.43, p<0.05)$ (Fig. 1). MOR and $D_{2} R$ availabilities in putamen were not associated in either group.

The association between MOR and $\mathrm{D}_{2} \mathrm{R}$ availabilities in the ventral striatum was recovered $(r=.62, p<0.05)$ among obese subjects following the surgery-induced weight loss (mean total weight loss $25.0 \pm 8.2 \mathrm{~kg}$ and $22.1 \pm 6.1 \%$ ) (Figs. 1 and 2). There was no difference between the two surgical procedures in receptor availabilities or the association between receptors before or after surgery.

In the volumetric analysis of striatal areas, there was no significant difference in striatal volumes between preoperative obese subjects and controls in any ROI ( $p s>$ 0.05 ; Table 2). Weight loss did not influence volumes in any region $(p s>0.05$; Table 2$)$.

\section{Discussion}

Our main finding was that opioid-dopamine interaction is recovered by bariatric surgery and concomitant weight loss. No change in striatal volumes was observed postoperatively. Dysfunctional opioid-dopamine interaction in the ventral striatum is associated with an obese phenotype and may mediate excessive energy uptake, and we have reported earlier that MOR levels return to normal after weight $\operatorname{loss}^{34}$. Behaviorally this is in line with previous studies, showing improved satiety and lowered appetite after bariatric surgery $^{41,42}$. We have previously shown that striatal opioid and dopamine systems are coupled in non-obese but not in obese subjects $^{29}$. In the normal-weight subjects, the interaction was strongest in the ventral striatum, but also significant in the dorsal caudate. Growing evidence indicates that MOR and $D_{2} R$ are expressed in the same striatal neurons ${ }^{26,43}$. The interaction between these receptor systems is likely crucial in regulating appetite, because it breaks down in the striatum in the obese subjects, while 


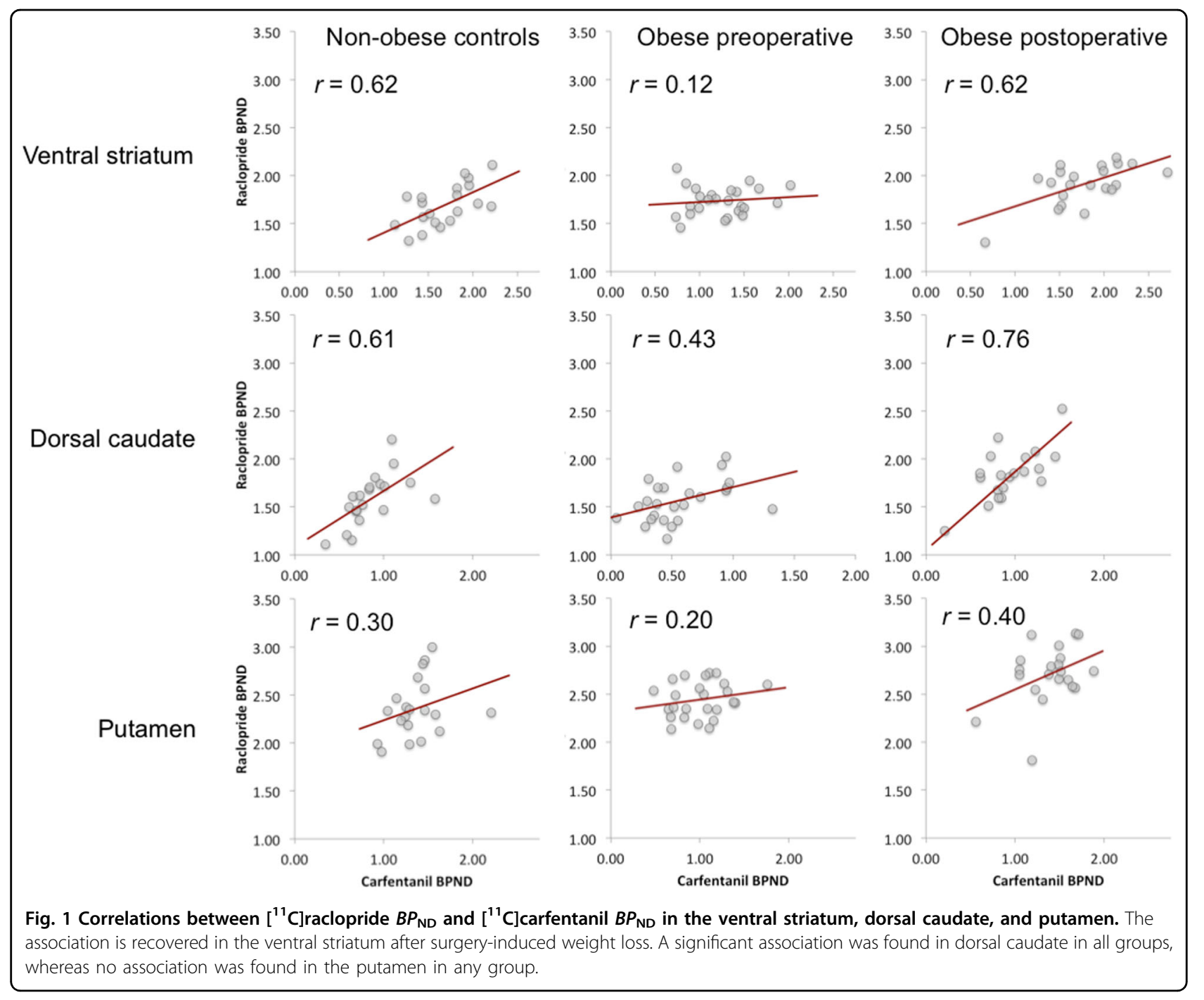

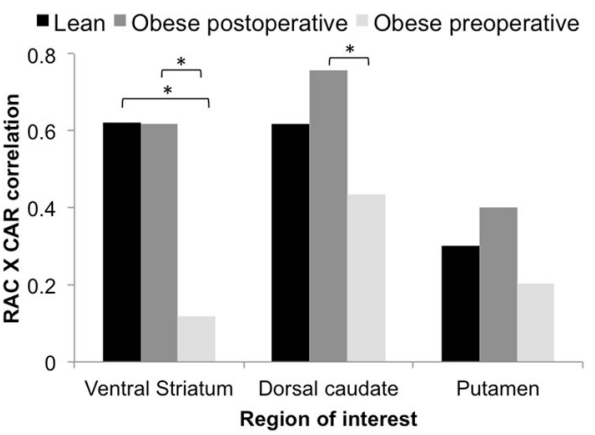

Fig. 2 Mean correlations between $\left[{ }^{11} \mathrm{C}\right]$ raclopride $B P_{\mathrm{ND}}$ and $\left[{ }^{11} \mathrm{C}\right]$ carfentanil $B P_{N D}$ in non-obese and obese subjects before and after surgery. Asterisks denote significant between-groups differences. association in the dorsal caudate remains intact. This might explain unaltered $D_{2} R$ levels in obesity: although obesitydependent dysfunction in the dopaminergic system is shown in numerous animal studies, it may be mediated through MOR-dependent mechanisms without having any effect on the actual number of $D_{2} R$ proteins. Even if the amount of $D_{2} R$ protein stays the same in obesity, decoupling of MOR and $D_{2} R$ in the striatum may cause altered dopaminergic functions.

MORs are co-localized with $\mathrm{D}_{2} \mathrm{Rs}$ in striosomes ${ }^{44}$. Dopaminergic neurons in the striosomes project directly to the ventral tegmental area (VTA) and substantia nigra, whereas neurons projecting to GABAergic neurons are distributed in the matrix compartment ${ }^{45}$. Pathways projecting from striosomes back to the midbrain exert disinhibitory control over the dopaminergic 
Table 2 Volumes of striatal areas.

\begin{tabular}{lccccc}
\hline & Obese preoperative $(\boldsymbol{N}=\mathbf{2 5})$ & $\boldsymbol{p}^{\mathbf{A}}$ & Obese postoperative $(\boldsymbol{N}=\mathbf{2 1})$ & $\boldsymbol{p}^{\mathbf{B}}$ & Healthy control subjects $(\boldsymbol{N}=\mathbf{2 0})$ \\
\hline Ventral striatum $\left(\mathrm{mm}^{3}\right)$ & $890 \pm 103$ & 0,30 & $930 \pm 131$ & 0,40 & $925 \pm 120$ \\
Dorsal caudate $\left(\mathrm{mm}^{3}\right)$ & $6623 \pm 812$ & 0,99 & $6634 \pm 918$ & 0,20 & $6319 \pm 582$ \\
Putamen $\left(\mathrm{mm}^{3}\right)$ & $9054 \pm 966$ & 0,81 & $8995 \pm 982$ & 0,15 & $8550 \pm 1116$ \\
\hline
\end{tabular}

Data are presented as mean \pm SD. $P$ value columns indicate differences between preoperative and postoperative measurements of the obese subjects $\left(p^{A}\right)$ and between preoperative obese subjects and control subjects $\left(p^{\mathrm{B}}\right)$.

neurons ${ }^{46}$, thus having a direct influence on the reward functions. These neurons are under direct opioidergic control $^{47}$. Accordingly, endogenous opioids disinhibit the neurons projecting from the patches to the midbrain (i.e., disinhibiting the disinhibiting neurons), and in this way increase dopaminergic firing in VTA. The rewarding effects of opioids are dependent on the MORs located in the striosomes ${ }^{48}$

Based on the observation that dopamine release caused by opioids in the striatum is dependent on the MORs in the striosomes in mice ${ }^{48}$, we hypothesize that aberrant opioid function in obese humans might lead to diminished dopamine release caused by eating. When obese subjects lose weight, the interaction between MOR and $D_{2} R$ is reverted. This further supports the notion that the interaction between these receptor systems is a normal state. The dysfunction of opioiddopamine interaction in the ventral striatum might be an important factor underlying overeating, and thus a feasible target for pharmacological and behavioral interventions. This has already been noted in pharmacological studies. MOR antagonist naltrexone therapy alone does not lead to significant weight loss, but promising results are obtained when it is coupled with bupropion (a dopamine and norepinephrine reuptake inhibitor $)^{49-52}$. Combination therapy of naltrexone and bupropion has been approved by FDA and EMA for weight management in adults ${ }^{53}$ and a certain amount of obese patients achieve significant weight loss ${ }^{54-56}$. The favorable effect of the combination therapy may be due to the tight coupling of $\mathrm{MOR}$ and $\mathrm{D}_{2} \mathrm{R}$. Moreover, the better efficacy of the combination therapy over monotherapies underlines the complex pattern of neurotransmitter networks underlying overeating and suggests that both aspects of reward functions-wanting and liking, processes mediated by dopaminergic and opioidergic systems, respectively ${ }^{57}$-have to be taken care of in order to treat obese patients.

This study has certain limitations. Only female subjects were studied, and the results may not be generalizable to male subjects. It was not possible to differentiate the combined effects of postoperative weight loss and altered gut anatomy and function. Altered neuroreceptor interaction may be due to the changes in gut hormones but also due to reduced intake of palatable foods. Further studies are needed to elucidate the sole effect of weight loss due to altered energy intake on the interaction of opioid and dopamine receptors by comparing the effects of weight loss by surgery versus dieting.

\section{Conclusions}

Obesity is associated with disrupted opioid-dopamine interaction in the ventral striatum, but this is recovered by weight loss after bariatric surgery. The dysfunction of opioid-dopamine interaction might be an important factor underlying overeating.

\begin{abstract}
Acknowledgements
The study was conducted within the Finnish Centre of Excellence in Cardiovascular and Metabolic Diseases supported by the Academy of Finland (grants \#251125, \#121031, \#304385), Sigrid Juselius Foundation, University of Turku, Turku University Hospital, and Åbo Akademi University. HKK was supported by personal grants from The Finnish Diabetes Research Foundation and The National Graduate School of Clinical Investigation. The funders had no role in study design, data collection, and analysis, decision to publish, or preparation of the manuscript. The authors thank the staff of the Turku PET Centre for assistance in the PET imaging. Janne Isojärvi is warmly thanked for conducting the FreeSurfer analysis in this study. Special thanks goes to research nurse Mia Koutu.
\end{abstract}

\begin{abstract}
Author details
${ }^{1}$ Turku PET Centre, Turku University Hospital and University of Turku, Turku, Finland. ${ }^{2}$ Institute of Mental Health Research, University of Ottawa, Ottawa, ON, Canada. ${ }^{3}$ Department of Surgery, University of Turku, Turku, Finland. ${ }^{4}$ Division of Digestive Surgery and Urology, Turku University Hospital, Turku, Finland. ${ }^{5}$ Department of Endocrinology, Turku University Hospital, Turku, Finland. ${ }^{6}$ Department of Psychology, University of Turku, Turku, Finland
\end{abstract}

\section{Author contributions}

H.K.: acquired and analyzed PET data, wrote the manuscript. L.T.: analyzed PET data, wrote the manuscript. S.H.: produced the radiotracers, wrote the manuscript. P.S.: recruited the study subjects, wrote the manuscript. P.N.: designed the experiments, wrote the manuscript. L.N.: designed the experiments, wrote the manuscript.

\section{Conflict of interest}

The authors declare no competing interests.

\section{Publisher's note}

Springer Nature remains neutral with regard to jurisdictional claims in published maps and institutional affiliations.

Supplementary information The online version contains supplementary material available at https://doi.org/10.1038/s41398-021-01370-2. 
Received: 16 January 2021 Revised: 18 March 2021 Accepted: 6 April 2021 Published online: 01 May 2021

\section{References}

1. Morales, I. \& Berridge, K. C. 'Liking' and 'wanting' in eating and food reward: Brain mechanisms and clinical implications. Physiol. Behav. 227, 113152 (2020)

2. Stice, E. \& Burger, K. Neural vulnerability factors for obesity. Clin. Psychol. Rev. 68, 38-53 (2019).

3. Pecina, S. \& Smith, K. S. Hedonic and motivational roles of opioids in food reward: implications for overeating disorders. Pharmacol. Biochem. Behav. 97, 34-46 (2010).

4. Fields, H. L. \& Margolis, E. B. Understanding opioid reward. Trends Neurosci. 38, 217-225 (2015)

5. Cambridge, V. C. et al. Neural and behavioral effects of a novel mu opioid receptor antagonist in binge-eating obese people. Biol. Psychiatry 73, 887-894 (2013).

6. Nathan, P. J. et al. Opioid receptor modulation of hedonic taste preference and food intake: a single-dose safety, pharmacokinetic, and pharmacodynamic investigation with GSK1521498, a novel mu-opioid receptor inverse agonist. J. Clin. Pharmacol. 52, 464-474 (2012).

7. Burghardt, P. R., Rothberg, A. E., Dykhuis, K. E., Burant, C. F. \& Zubieta, J. K. Endogenous opioid mechanisms are implicated in obesity and weight loss in humans. J. Clin. Endocrinol. Metab. 100, 3193-3201 (2015).

8. Karlsson, H. K. et al. Obesity is associated with decreased $\mu$-opioid but unaltered dopamine D2 receptor availability in the brain. J. Neurosci. 35, 3959-3965 (2015).

9. Stice, E., Yokum, S., Burger, K. S., Epstein, L. H. \& Small, D. M. Youth at risk for obesity show greater activation of striatal and somatosensory regions to food. J. Neurosci. 31, 4360-4366 (2011).

10. Salamone, J. D. \& Correa, M. Dopamine and food addiction: lexicon badly needed. Biol. Psychiatry 73, e15-e24 (2013).

11. Volkow, N. D. et al. Low dopamine striatal D2 receptors are associated with prefrontal metabolism in obese subjects: possible contributing factors. Neurolmage 42, 1537-1543 (2008).

12. Lee, $Y$. et al. Lower dopamine tone in the striatum is associated with higher body mass index. Eur. Neuropsychopharmacol. 28, 719-731 (2018).

13. Eisenstein, S. A. et al. A comparison of D2 receptor specific binding in obese and normal-weight individuals using PET with (N-[(11)C]methyl)benperidol. Synapse 67, 748-756 (2013).

14. Horstmann, A., Fenske, W. K. \& Hankir, M. K. Argument for a non-linear relationship between severity of human obesity and dopaminergic tone. Obes. Rev. 16, 821-830 (2015).

15. Dang, L. C. et al. Associations between dopamine D2 receptor availability and BMI depend on age. Neurolmage 138, 176-183 (2016).

16. Le Merrer, J., Becker, J. A., Befort, K. \& Kieffer, B. L. Reward processing by the opioid system in the brain. Physiol. Rev. 89, 1379-1412 (2009).

17. Colasanti, A. et al. Endogenous opioid release in the human brain reward system induced by acute amphetamine administration. Biol. Psychiatry $\mathbf{7 2}$ 371-377 (2012)

18. Soderman, A. R. \& Unterwald, E. M. Cocaine-induced mu opioid receptor occupancy within the striatum is mediated by dopamine D2 receptors. Brain Res. 1296, 63-71 (2009).

19. Mick, l. et al. Amphetamine induced endogenous opioid release in the human brain detected with $[(1)(1) C]$ carfentanil PET: replication in an independent cohort. Int. J. Neuropsychopharmacol. 17, 2069-2074 (2014).

20. Jalabert, M. et al. Neuronal circuits underlying acute morphine action on dopamine neurons. Proc. Natl Acad. Sci. USA 108, 16446-16450 (2011).

21. Volkow, N. D. \& Wise, R. A. How can drug addiction help us understand obesity? Nat. Neurosci. 8, 555-560 (2005).

22. Spanagel, R., Herz, A. \& Shippenberg, T. S. Opposing tonically active endogenous opioid systems modulate the mesolimbic dopaminergic pathway. Proc. Natl Acad. Sci. USA 89, 2046-2050 (1992).

23. Kalivas, P. W. Neurotransmitter regulation of dopamine neurons in the ventral tegmental area. Brain Res. Rev. 18, 75-113 (1993).

24. Rada, P., Barson, J. R., Leibowitz, S. F. \& Hoebel, B. G. Opioids in the hypothalamus control dopamine and acetylcholine levels in the nucleus accumbens. Brain Res. 1312, 1-9 (2010).

25. Chartoff, E. H. \& Connery, H. S. It's MORe exciting than mu: crosstalk between mu opioid receptors and glutamatergic transmission in the mesolimbic dopamine system. Front. Pharmacol. 5, 116 (2014).
26. Margolis, E. B. \& Hjelmstad, G. O. Direct bidirectional mu-opioid control of midbrain dopamine neurons. J. Neurosci. 34, 14707-14716 (2014).

27. Yeomans, M. R. \& Gray, R. W. Opioid peptides and the control of human ingestive behaviour. Neurosci. Biobehav. Rev. 26, 713-728 (2002).

28. Taber, M. T., Zernig, G. \& Fibiger, H. C. Opioid receptor modulation of feedingevoked dopamine release in the rat nucleus accumbens. Brain Res. $\mathbf{7 8 5}$, 24-30 (1998).

29. Tuominen, L. et al. Aberrant mesolimbic dopamine-opiate interaction in obesity. Neurolmage 122, 80-86 (2015).

30. Adams, T. D. et al. Weight and metabolic outcomes 12 years after gastric bypass. N. Engl. J. Med. 377, 1143-1155 (2017).

31. Bryant, E. J., Malik, M. S., Whitford-Bartle, T. \& Waters, G. M. The effects of bariatric surgery on psychological aspects of eating behaviour and food intake in humans. Appetite 150, 104575 (2020).

32. de Weijer, B. A. et al. Striatal dopamine receptor binding in morbidly obese women before and after gastric bypass surgery and its relationship with insulin sensitivity. Diabetologia 57, 1078-1080 (2014).

33. Steele, K. E. et al. Alterations of central dopamine receptors before and after gastric bypass surgery. Obes. Surg. 20, 369-374 (2010).

34. Karlsson, H. K. et al. Weight loss after bariatric surgery normalizes brain opioid receptors in morbid obesity. Mol. Psychiatry 21, 1057-1062 (2016).

35. Dunn, J. P. et al. Decreased dopamine type 2 receptor availability after bariatric surgery: preliminary findings. Brain Res. 1350, 123-130 (2010).

36. Farde, L., Hall, H., Ehrin, E. \& Sedvall, G. Quantitative analysis of D2 dopamine receptor binding in the living human brain by PET. Science 231, 258-261 (1986).

37. Frost, J. J. et al. Imaging opiate receptors in the human brain by positron tomography. J. Computer Assist. Tomogr. 9, 231-236 (1985).

38. Gunn, R. N., Lammertsma, A. A., Hume, S. P. \& Cunningham, V. J. Parametric imaging of ligand-receptor binding in PET using a simplified reference region model. Neurolmage 6, 279-287 (1997).

39. Tzourio-Mazoyer, $\mathrm{N}$. et al. Automated anatomical labeling of activations in SPM using a macroscopic anatomical parcellation of the MNI MRI singlesubject brain. Neurolmage 15, 273-289 (2002).

40. Eickhoff, S. B. et al. A new SPM toolbox for combining probabilistic cytoarchitectonic maps and functional imaging data. Neurolmage 25, 1325-1335 (2005).

41. Morinigo, R. et al. Glucagon-like peptide-1, peptide $Y Y$, hunger, and satiety after gastric bypass surgery in morbidly obese subjects. J. Clin. Endocrinol. Metab. 91, 1735-1740 (2006).

42. Karamanakos, S. N., Vagenas, K., Kalfarentzos, F. \& Alexandrides, T. K. Weight loss, appetite suppression, and changes in fasting and postprandial ghrelin and peptide-YY levels after RouX-en-Y gastric bypass and sleeve gastrectomy: a prospective, double blind study. Ann. Surg. 247, 401-407 (2008).

43. Ambrose, L. M., Unterwald, E. M. \& Van Bockstaele, E. J. Ultrastructural evidence for co-localization of dopamine D2 and micro-opioid receptors in the rat dorsolateral striatum. Anat. Rec. Part A 279, 583-591 (2004).

44. Herkenham, M. \& Pert, C. B. Mosaic distribution of opiate receptors, parafascicular projections and acetylcholinesterase in rat striatum. Nature 291, 415-418 (1981)

45. Watabe-Uchida, M., Zhu, L., Ogawa, S. K., Vamanrao, A. \& Uchida, N. Wholebrain mapping of direct inputs to midbrain dopamine neurons. Neuron 74, 858-873 (2012).

46. Fujiyama, F., Takahashi, S. \& Karube, F. Morphological elucidation of basal ganglia circuits contributing reward prediction. Front. Neurosci. 9, 6 (2015).

47. Banghart, M. R., Neufeld, S. Q., Wong, N. C. \& Sabatini, B. L. Enkephalin disinhibits $\mathrm{Mu}$ opioid receptor-rich striatal patches via delta opioid receptors. Neuron 88, 1227-1239 (2015).

48. Cui, Y. et al. Targeted expression of mu-opioid receptors in a subset of striatal direct-pathway neurons restores opiate reward. Nat. Neurosci. 17, 254-261 (2014)

49. Lee, M. W. \& Fujioka, K. Naltrexone for the treatment of obesity: review and update. Expert Opin. Pharmacother. 10, 1841-1845 (2009).

50. Greenway, F. L. et al. Effect of naltrexone plus bupropion on weight loss in overweight and obese adults (COR-I): a multicentre, randomised, double-blind, placebo-controlled, phase 3 trial. Lancet 376, 595-605 (2010).

51. Billes, S. K., Sinnayah, P. \& Cowley, M. A. Naltrexone/bupropion for obesity: an investigational combination pharmacotherapy for weight loss. Pharmacol. Res. 84, 1-11 (2014). 
52. Smith, S. R. et al. Combination therapy with naltrexone and bupropion for obesity reduces total and visceral adiposity. Diabetes, Obes. Metab. 15, 863-866 (2013).

53. Sherman, M. M., Ungureanu, S. \& Rey, J. A. Naltrexone/Bupropion ER (Contrave): newly approved treatment option for chronic weight management in obese adults. Pharmacy Ther. 41, 164-172 (2016).

54. Fujioka, K. et al. The relationship between early weight loss and weight loss at 1 year with naltrexone ER/bupropion ER combination therapy. Int. J. Obes. $\mathbf{4 0}$ 1369-1375 (2016).
55. Mullally, J. A. et al. Weight loss response to naltrexone/bupropion is modulated by the Taq1A genetic variant near DRD2 (rs1800497): a pilot study. Diabetes Obesity Metabol. 23, 850-853 (2020).

56. Onakpoya, I. J., Lee, J. J., Mahtani, K. R., Aronson, J. K. \& Heneghan, C. J. Naltrexone-bupropion (Mysimba) in management of obesity: a systematic review and meta-analysis of unpublished clinical study reports. Br. J. Clin. Pharmacol. 86, 646-667 (2020).

57. Berridge, K. C. \& Kringelbach, M. L. Pleasure systems in the brain. Neuron $\mathbf{8 6}$, 646-664 (2015). 